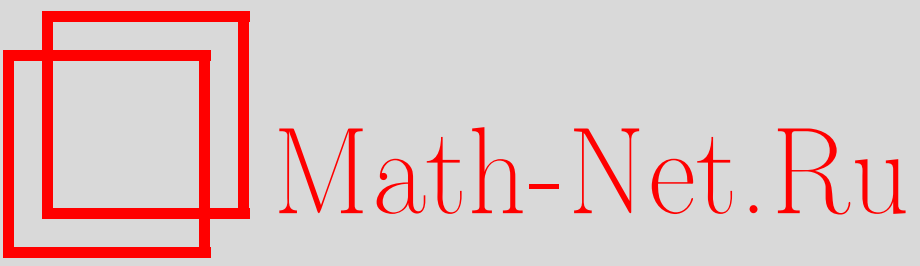

3. М. Шибзухов, Минимизации робастных оценок сумм параметризованных функций, Итоги науки и техн. Сер. Соврем. мат. и ее прил. Темат. обз., 2019, том 166, 95-109

DOI: https://doi.org/10.36535/0233-6723-2019-166-95-109

Использование Общероссийского математического портала Math-Net.Ru подразумевает, что вы прочитали и согласны с пользовательским соглашением

http://www.mathnet.ru/rus/agreement

Параметры загрузки :

IP : 44.207 .124 .84

26 апреля 2023 г., 16:44:08 


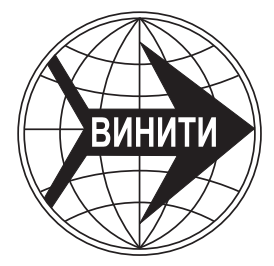

ИТОГИ НАУКИ И ТЕХНИКИ.

Современная математика и ее приложения.

Тематические обзоры.

Том 166 (2019). С. 95-109

DOI: $10.36535 / 0233-6723-2019-166-95-109$

УДК 519.7

\title{
МИНИМИЗАЦИИ РОБАСТНЫХ ОЦЕНОК СУММ ПАРАМЕТРИЗОВАННЫХ ФУНКЦИЙ
}

\author{
(c) 2019 г. $\quad$ 3. М. ШИБЗУХОВ
}

\begin{abstract}
АннотАция. Рассматривается робастный подход к построению алгоритмов машинного обучения, основанный на минимизации робастных конечных сумм параметризованных функций. Он основывается на применении конечных робастных дифференцируемых агрегирующих функций суммирования, которые являются устойчивыми по отношению к выбросам.
\end{abstract}

Ключевые слова: робастный алгоритм, нейронная сеть, усредняющая агрегирующая функция, итеративное перевзвешивание.

\section{MINIMIZING ROBUST ESTIMATES \\ OF SUMS OF PARAMETERIZED FUNCTIONS}

\section{(C) 2019 Z. M. SHIBZUKHOV}

Abstract. We discuss the robust approach to constructing machine learning algorithms based on minimizing robust finite sums of parameterized functions. This algorithm is based on finite robust differentiable aggregation summation functions, which are stable with respect to outliers.

Keywords and phrases: robust algorithm, neural network, averaging aggregation function, iterative reweighing.

AMS Subject Classification: 68T05

1. Введение. Значительную часть задач классификации и регрессии можно свести к задаче минимизации конечных сумм параметризованных функций:

$$
\mathcal{Q}(\mathbf{w})=\sum_{k=1}^{N} v_{k} \ell_{k}(\mathbf{w}),
$$

где $\ell_{1}(\mathbf{w}), \ldots, \ell_{N}(\mathbf{w})$ - неотрицательные базисные функции, $\mathbf{w}$ - вектор неизвестных параметров, $\mathbf{w} \in \mathbf{W} \subseteq \mathbb{R}^{m}, v_{1}, \ldots, v_{N} \geqslant 0$ - заданные неотрицательные числовые веса. Типичные примерыэто обычная сумма

$$
\mathcal{Q}(\mathbf{w})=\sum_{k=1}^{N} \ell_{k}(\mathbf{w})
$$

или среднее арифметическое

$$
\mathcal{Q}(\mathbf{w})=\frac{1}{N} \sum_{k=1}^{N} \ell_{k}(\mathbf{w}) .
$$

\footnotetext{
Работа выполнена при поддержке Российского фонда фундаментальных исследований (проект № 18-01-00050).
} 
Оптимальный набор параметров $\mathbf{w}^{*}$ должен минимизировать целевую функцию $\mathcal{Q}$ :

$$
\mathcal{Q}\left(\mathbf{w}^{*}\right)=\min _{\mathbf{w} \in \mathbf{W}} \mathcal{Q}(\mathbf{w}) .
$$

Для более предметного обсуждения приведем типичные постановки задач и необходимые обозначения.

Задача регрессии. В задаче регрессии восстанавливается неизвестная зависимость на основе параметрической модели $y=f(\mathbf{x}, \mathbf{w})$. Функции потерь для $k$-го прецедента имеет вид

$$
\ell_{k}(\mathbf{w})=\varrho\left(f\left(\mathbf{x}_{k}, \mathbf{w}\right)-y_{k}\right),
$$

где $\varrho(r)$ - такая неотрицательная квазивыпуклая функция, что $\varrho(0)=\min \varrho(r)$.

Задача классификации с двумя классами. В задаче классификации с двумя классами восстанавливается зависимость на основе параметрической модели $y=\operatorname{sign} f(\mathbf{x}, \mathbf{w})$. Функция потерь для $k$-го прецедента имеет вид

$$
\ell_{k}(\mathbf{w})=\varrho\left(y_{k} f\left(\mathbf{x}_{k}, \mathbf{w}\right)\right)
$$

где $\varrho(r)$ - такая неотрицательная монотонно убывающая функция, что $\lim _{r \rightarrow \infty} \varrho(r)=0$.

Задача классификации с q классами. В задаче классификации с $q$ классами восстанавливается зависимость в форме

$$
y=\arg \max _{j=1, \ldots, q} f_{j}(\mathbf{x}, \mathbf{w})
$$

где $f_{j}(\mathbf{x}, \mathbf{w})$ - параметрическая функция вычисления оценки за $j$-й класс. Функция потерь для $k$-го прецедента имеет вид

$$
\ell_{k}(\mathbf{w})=\varrho\left(r_{1, k}(\mathbf{w}), \ldots, r_{q-1, k}(\mathbf{w})\right),
$$

где $\varrho\left(r_{1}, \ldots, r_{q}\right)$ - неотрицательная монотонно убывающая функция, стремящаяся к нулю при $r_{1} \rightarrow \infty, \ldots, r_{q} \rightarrow \infty$. Значения $r_{1, k}(\mathbf{w}), \ldots, r_{q-1, k}(\mathbf{w})$ вычисляются по $f_{1}\left(\mathbf{x}_{k}, \mathbf{w}\right), \ldots, f_{q}\left(\mathbf{x}_{k}, \mathbf{w}\right)$ при помощи следующего правила:

$$
r_{1}, \ldots, r_{q-1}= \begin{cases}f_{1}-f_{2}, \ldots, f_{1}-f_{q}, & \text { если } y_{k}=1, \\ f_{j}-f_{1}, \ldots, f_{j}-f_{j-1}, f_{j}-f_{j+1}, \ldots, f_{j}-f_{q}, & \text { если } 1<y_{k}<q-1, \\ f_{q}-f_{1}, \ldots, f_{q}-f_{q-1}, & \text { если } y_{k}=q-1 .\end{cases}
$$

Принцип поиска оптимальных параметров и проблема выбросов. Однако если распределение значений базисных функций на обучающих данных содержит выбросы (например, из-за выбросов в самих данных), то минимизация $\mathcal{Q}(\mathbf{w})$, как правило, приводит к искажению $\mathbf{w}^{*}$. Это происходит из-за того, что арифметическая сумма и среднее арифметическое не являются устойчивыми по отношению к выбросам.

Для преодоления вляния выбросов можно было бы подобрать такие веса $v_{1}, \ldots, v_{N}$, которые принимают значения, близкие к нулю, для прецедентов, связанных с выбросами. Однако эта задача сопоставима по сложности с задачей идентификации самих выбросов. Простой подход, когда сначала решается задача минимизации (2) или (3), а затем по величине ошибок или значений базисных функций идентифицируются сами выбросы, работает далеко не всегда.

Классический подход к решению проблемы выбросов. Достаточно распространенный подход к преодолению влияния выбросов основан на попытке построения базисных функций, которые растут достаточно медленно с ростом ошибки или отступа, чтобы преодолеть влияние выбросов в данных. Приведем примеры построения таких функций путем выбора подходящей функции $\varrho$. 
Задача регрессии. В задаче регрессии используются такие выпуклые функции $\varrho(r)$, которые растут существенно медленнее, чем $r^{2}$, или же квазивыпуклые $\varrho(r)$, которые являются ограниченными, например,

(1) функция

$$
\varrho(r)= \begin{cases}0, & \text { если }|r|<c, \\ |r|-c, & \text { если }|r| \geqslant c ;\end{cases}
$$

(2) функция Хьюбера:

$$
\varrho(r)= \begin{cases}\frac{r^{2}}{2 c}, & \text { если }|r|<c, \\ |r|-\frac{c}{2}, & \text { если }|r| \geqslant c ;\end{cases}
$$

(3) функция Тьюки:

$$
\varrho(r)= \begin{cases}\frac{c^{2}}{6}\left[1-\left(1-\left(\frac{r}{c}\right)^{2}\right)^{3}\right], & \text { если }|r| \leqslant c, \\ \frac{c^{2}}{6}, & \text { если }|r|>c ;\end{cases}
$$

(4) функция Эндрю:

$$
\varrho(r)= \begin{cases}2 c, & \text { если }|r|>c, \\ c\left(1-\cos \left(\frac{\pi r}{c}\right)\right), & \text { если }|r| \leqslant c .\end{cases}
$$

Задача классификачии с двумя классами. В задаче классификации на два класса используются такие функции $\varrho(r)$, которые быстро убывают на $[0, \infty)$ и ассимптотически убывают на $(-\infty, 0]$ не быстрее, чем линейная функция, например,

(1) функция Хинжа:

$$
\varrho(r)= \begin{cases}c-r, & \text { если } r<c, \\ 0, & \text { если } r \geqslant c ;\end{cases}
$$

(2) функция логистической регрессии:

$$
\varrho(r)=\frac{1}{c} \ln \left(1+e^{-c r}\right)
$$

(3) сигмоидальная функция:

$$
\varrho(r)=\left(1+e^{c r}\right)^{-1}
$$

Задача классификации с q классами. В задаче классификации на $q$ класса используются такие функции $\varrho\left(r_{1}, \ldots, r_{q}\right)$, которые быстро убывают на $[0, \infty)^{q}$ и асимптотически убывают на $(-\infty, 0]^{q}$ не быстрее, чем линейная функция, например,

(1) функция мягкого минимума:

$$
\varrho\left(r_{1}, \ldots, r_{q}\right)=-\ln \left(\alpha_{1} e^{-r_{1}}+\cdots+\alpha_{1} e^{-r_{q}}\right),
$$

где $\alpha_{1}, \ldots, \alpha_{q}>0$;

(2) сумма скалярных функций потерь:

$$
\varrho\left(r_{1}, \ldots, r_{q}\right)=\alpha_{1} \varrho_{1}\left(r_{1}\right)+\cdots+\alpha_{1} \varrho_{q}\left(r_{q}\right),
$$

где $\varrho_{1}, \ldots, \varrho_{1}-$ скалярные функции потерь, которые применяются в задаче классификации с двумя классами. 
Оценка робастности классического подхода. В рамках указанного выше подхода для ряда постановок задач функция $\ell_{k}$ имеет вид $\ell_{k}(\mathbf{w})=\varrho\left(r_{k}(\mathbf{w})\right)$. В задачах регрессии $r_{k}(\mathbf{w})$ - оценка величины ошибки, в задачах классификации для двух классов $r_{k}(\mathbf{w})$ - оценка величины отступа. Это позволяет рассмотрение проблемы выбросов в распределении значений базисных функций свести к рассмотрению проблемы выбросов в распределении ошибок или отступов, соответственно.

Для того чтобы оценить возможности по преодолению выбросов, рассмотрим следующее соотношение (в предположении, что $\varrho$ дифференцируема):

$$
|\varrho(r+\Delta)-\varrho(r)|=\left|\varrho^{\prime}(\tilde{r})\right| \Delta,
$$

где $\Delta$ - величина искажения, $\tilde{r}$ - значение, заключенное между $r$ и $r+\Delta$.

Для задач классификации для $q$ классов $\ell_{k}(\mathbf{w})=\varrho\left(r_{1, k}(\mathbf{w}), \ldots, r_{q-1, k}(\mathbf{w})\right)$. Следующее неравенство лежит в основе оценки возможности классического подхода по преодолению выбросов:

$$
\left|\varrho\left(r_{1}+\Delta_{1}, \ldots, r_{q-1}+\Delta_{q-1}\right)-\varrho\left(r_{1}, \ldots, r_{q-1}\right)\right|=\left|\varrho^{\prime}(\tilde{\mathbf{r}})\right| \cdot\left\|\left(\Delta_{1}, \ldots, \Delta_{q-1}\right)\right\|,
$$

где вектор $\tilde{\mathbf{r}}$ лежит в $(q-1)$-мерном сегменте, образованном декартовым произведением сегментов с границами $r_{1}$ и $r_{1}+\Delta_{1}, \ldots, r_{q-1}$ и $r_{q-1}+\Delta_{q-1}$, соответственно.

Здесь возможны два основных случая. Если $\left|\varrho^{\prime}(r)\right| \leqslant C$, а больших выбросов $(\Delta \gg 1)$ относительно немного, то возможно преодолеть их влияние или по крайней мере идентифицировать их. Однако если больших выбросов много, то этот подход уже не работает, и в таких случаях применяют такие функции $\varrho(r)$, что $|\varrho(r)| \leqslant C$. В этом случае сходимость алгоритмов поиска $\mathbf{w}^{*}$ существенно зависит от выбора начального приближения $\mathbf{w}_{0}$ : если оно окажется далеко от $\mathbf{w}^{*}$, то поиск при помощи градиентных методов не увенчается успехом из-за того, что производная $\varrho^{\prime}(r)$ сремится к нулю, когда $|r| \rightarrow \infty$ и, как следствие, будет найден такой набор параметров, при котором ошибки окажутся очень большими по модулю, а отступы-отрицательными числами с большими значениями по модулю.

Использование медианы и усеченного среднего арифметического. Возникают ситуации, когда невозможно подобрать функцию $\varrho$ так, чтобы с ее помощью можно было бы подавить влияние большого числа (вплоть до 50\%) выбросов. Тогда на первый план выступает второе препятствие на пути преодоления выбросов: среднее арифметическое не является устойчивым по отношению к выбросам. Поэтому для разрешения проблемы выбросов целесообразно использовать такие оценки среднего значения, которые были бы более устойчивыми по отношению к выбросам. Так, вместо среднего арифметического стали использовать медиану для робастной оценки среднего значения

$$
\mathcal{Q}(\mathbf{w})=\operatorname{med}_{k=1, \ldots N} \ell_{k}(\mathbf{w}),
$$

а вместо обычной суммы - урезанную сумму:

$$
\mathcal{Q}(\mathbf{w})=\sum_{k=1}^{N-p} \ell_{(k)}(\mathbf{w}),
$$

которую можно заменить на урезанное среднее арифметическое:

$$
\mathcal{Q}(\mathbf{w})=\frac{1}{N-p} \sum_{k=1}^{N-p} \ell_{(k)}(\mathbf{w}),
$$

где $z_{(1)}, \ldots, z_{(N)}$ - последовательность чисел, которая получается в результате упорядочивания по возрастанию исходной последовательности $z_{1}, \ldots, z_{N}$. Например, для построения робастной регрессии с $\ell_{k}(\mathbf{w})=\left(f\left(\mathbf{x}_{k}, \mathbf{w}\right)-y_{k}\right)^{2}$ были предложены метод LMedS (least median squares) и метод LTS (least trimed squares; cм. $[13,14]$ ). Минимизация таких оценок при наличии выбросов в данных (вплоть до 50\%) для конечных сумм или средних часто позволяет идентифицировать выбросы или даже находить адекватные оценки для $\mathbf{w}^{*}$. Однако алгоритмы минимизации $\mathcal{Q}(\mathbf{w})$ включают комбинаторную составляющую в виде поиска $\mathbf{w}^{*}$ по подвыборкам. 
Применение алгоритмов градиентного типа невозможно, так как медиана и усеченное среднее арифметическое не являются непрерывно дифференцируемыми: их частные производные являются разрывными функциями, имеющими такие свойства, которые существенно осложняют их применение для обучения нейронных сетей.

Отметим еще один подход, основанный на использовании цензурированной суммы

$$
\mathcal{Q}(\mathbf{w})=\sum_{k=1}^{N-p} \ell_{(k)}(\mathbf{w})+p \bar{\ell}(\mathbf{w})
$$

или цензурированного среднего арифметического

$$
\mathcal{Q}(\mathbf{w})=\frac{1}{N} \sum_{k=1}^{N-p} \ell_{(k)}(\mathbf{w})+\frac{p}{N} \bar{\ell}(\mathbf{w}),
$$

где $\bar{\ell}(\mathbf{w})$ - функция, вычисляющая некоторое пороговое значение, так что слагаемые, которые превышают заданное пороговое значение $\bar{\ell}(\mathbf{w})$, заменяются на $\bar{\ell}(\mathbf{w})$.

В настоящей работе предлагается общий подход, основанный на применении робастных непрерывно дифференцируемых оценок среднего значения. Это позволяет строить градиентные процедуры обучения, не требующие привлечение комбинаторного поиска по подвыборкам.

2. Функции М-среднего. Использование медианы вместо среднего арифметического делает практически невозможным использование традиционных и хорошо изученных процедур минимизации градиентного типа. Для решения этой проблемы введем обобщающее понятие М-средних, среди которых можно выделить непрерывно дифференцируемые и в определенной степени устойчивые по отношению к выбросам.

Функции М-среднего можно определить, взяв за основу определение усредняющих агрегирующих функций, основанных на штрафах (см. [4,5]). Для этого используем штрафнъе функиии, которые имеют следующий вид:

$$
P\left(z_{1}, \ldots, z_{N}, u\right)=\sum_{k=1}^{N} \rho\left(z_{k}-u\right),
$$

где $\rho(x)$ - выпуклая функция, которая имеет единственный минимум в нуле.

Введем обозначение

$$
\mathbf{M}_{z_{1} \ldots z_{N}}=\left\{u: P\left(z_{1}, \ldots, z_{N}, u\right)=P_{\min }\right\} .
$$

Функция М-среднего $\mathrm{M}_{\rho}\left\{z_{1}, \ldots, z_{N}\right\}$, основанная на функции несходства $\rho$, определяется следующим образом:

$$
\mathrm{M}_{\rho}\left\{z_{1}, \ldots, z_{N}\right\}=\arg \min _{u} P\left(z_{1}, \ldots, z_{N}, u\right)
$$

если $\mathbf{M}_{z_{1} \ldots z_{N}}-$ синглетон и

если $\mathbf{M}_{z_{1} \ldots z_{N}}$ - сегмент с концами $a$ и $b$.

$$
\mathrm{M}_{p}\left\{z_{1}, \ldots, z_{N}\right\}=\frac{a+b}{2},
$$

Приведем примеры семейств М-средних, которые включают в себя хорошо знакомые функции.

Семейство симметричных средних:

$$
\mathrm{M}^{\gamma}\left\{z_{1}, \ldots, z_{N}\right\}=\arg \min _{u} \sum_{k=1}^{N}\left|z_{k}-u\right|^{1+\gamma},
$$

где $0 \leqslant \gamma \leqslant 1$; при этом $\mathrm{M}^{0}$ - медиана, $\mathrm{M}^{1}$ - среднее арифметическое.

\section{Семейство несимметричных средних:}

$$
\mathrm{M}_{\alpha}^{\gamma}\left\{z_{1}, \ldots, z_{N}\right\}=\arg \min _{u} \sum_{k=1}^{N}\left|z_{k}-u\right|_{\alpha}^{1+\gamma},
$$

где $|u|_{\alpha}^{1+\gamma}=(\alpha-[u>0]) u|u|^{\gamma}, 0 \leqslant \gamma \leqslant 1$; при этом $\mathrm{M}_{\alpha}^{0}-\alpha$-квантиль, $\mathrm{M}_{\alpha}^{1}-\alpha$-экспектиль. 
Если функция $\rho$ имеет частные производные, то среднее значение $\bar{z}=\mathrm{M}_{\rho}\left\{z_{1}, \ldots, z_{N}\right\}$ является решением уравнения

$$
\sum_{k=1}^{N} \rho_{u}^{\prime}\left(z_{k}-\bar{z}\right)=0
$$

которое можно рассматривать в качестве определения функции М-среднего как неявной функции, если множество $\mathbf{M}_{z_{1} \ldots z_{N}}$ - синглетон.

Если $\rho$ является дважды дифференцируемой, то $\mathrm{M}_{\rho}\left\{z_{1}, \ldots, z_{N}\right\}$ имеет все частные производные:

$$
\frac{\partial \mathrm{M}_{\rho}}{\partial z_{k}}=\frac{\rho^{\prime \prime}\left(z_{k}-\bar{z}\right)}{\rho^{\prime \prime}\left(z_{1}-\bar{z}\right)+\cdots+\rho^{\prime \prime}\left(z_{N}-\bar{z}\right)},
$$

где $\bar{z}=\mathrm{M}_{\rho}\left\{z_{1}, \ldots, z_{N}\right\}$. Заметим, что

$$
\frac{\partial \mathrm{M}_{\rho}}{\partial z_{1}}+\cdots+\frac{\partial \mathrm{M}_{\rho}}{\partial z_{N}}=1
$$

Если $\rho$ - выпуклая функция, то также $\partial \mathrm{M}_{\rho} / \partial z_{k} \geqslant 0$.

2.1. Робастные М-средние. Для того, чтобы выяснить, в каких случаях функция М-среднего $\mathrm{M}_{\rho}$ может быть устойчивой по отношению к выбросам, рассмотрим следующее неравенство:

$$
\left|\mathrm{M}_{\rho}\left\{z_{1}, \ldots, z_{N}+\Delta\right\}-\mathrm{M}_{\rho}\left\{z_{1}, \ldots, z_{N}\right\}\right|=\frac{\rho^{\prime \prime}\left(\tilde{z}-u_{\tilde{z}}\right) \Delta}{\sum_{k=1}^{N-1} \rho^{\prime \prime}\left(z_{k}-u_{\tilde{z}}\right)+\rho^{\prime \prime}\left(\tilde{z}-u_{\tilde{z}}\right)}<\rho^{\prime \prime}\left(\tilde{z}-u_{\tilde{z}}\right) \Delta,
$$

где $\rho(r)$ - выпуклая функция, $\rho^{\prime \prime}(r)$ - непрерывная функция, $\Delta>0$-величина искажения, $\tilde{z} \in\left[z_{N}, z_{N}+\Delta\right], u_{\tilde{z}}=\mathrm{M}_{\rho}\left\{z_{1}, \ldots, z_{N-1}, \tilde{z}\right\}$.

Приведем примеры М-средних, которые являются дифференцируемыми и при достаточно малых $\varepsilon$ - устойчивыми по отношению к выбросам (в некоторых случаях вплоть до $50 \%$ ).

1. $\rho_{\varepsilon}(r)=\sqrt{\varepsilon^{2}+r^{2}}-\varepsilon$; при этом $\rho_{\varepsilon}^{\prime \prime}(r)=\varepsilon^{2} /\left(\varepsilon^{2}+r^{2}\right)^{3 / 2}<\varepsilon^{2} / r^{3}$, поэтому при $\Delta<r^{3}$ имеем $\rho_{\varepsilon}^{\prime \prime}(r) \Delta<\varepsilon^{2}$.

2. $\rho_{\varepsilon}(r)=|r|-\varepsilon \ln (\varepsilon+|r|)-\varepsilon \ln \varepsilon$; при этом $\rho_{\varepsilon}^{\prime \prime}(r)=\varepsilon /(\varepsilon+|r|)^{2}<\varepsilon / r^{2}$, поэтому при $\Delta<r^{2}$ имеем $\rho_{\varepsilon}^{\prime \prime}(r) \Delta<\varepsilon$.

Приведенные примеры показывают, что существуют дифференцируемые М-средние, устойчивые по отношению к выбросам при выборе достаточно малого значения параметра $\varepsilon$, которые можно рассматривать в качестве замены медианы.

В качестве замены $\alpha$-квантилей можно использовать М-средние, основанные на функции

$$
\rho_{\varepsilon, \alpha}(r)= \begin{cases}(1-\alpha) \rho_{\varepsilon}(r), & \text { если } r<0, \\ \frac{1}{2}\left(\alpha \rho_{\varepsilon}^{\prime}\left(0^{+}\right)+(1-\alpha) \rho_{\varepsilon}^{\prime}\left(0^{-}\right)\right), & \text {если } x=0, \\ \alpha \rho_{\varepsilon}(r), & \text { если } r>0 .\end{cases}
$$

2.2. Градиентные прочедуры для вычисления М-средних. Для вычисления М-среднего можно применить любой метод минимизации функции

$$
P(u)=P\left(z_{1}, \ldots, z_{N}, u\right)=\sum_{k=1}^{N} \rho\left(z_{k}-u\right) .
$$

Если существует производная $\rho_{u}^{\prime}(z-u)$, то для вычисления приближенного значения $\mathrm{M}_{\rho}\left\{z_{1}, \ldots, z_{N}\right\}$ можно использовать любой градиентный метод минимизации (9). Например, метод полного градиента:

$$
u_{t+1} \leftarrow u_{t}-\tau Q_{t}, \quad x \text { где } \quad Q_{t} \leftarrow \frac{1}{N} \sum_{k=1}^{N} \rho_{u}^{\prime}\left(z_{k}-u_{t}\right) .
$$


Для построения стохастического аналога, имеющего такой же порядок сходимости, можно воспользоваться схемой построения алгоритма SAG (см. [15]). В этой схеме величина $Q_{t}$ обновляется по правилу

$$
Q_{t}=\frac{1}{N} \sum_{k=1}^{N} Q_{t, k}
$$

а набор $\left\{Q_{t+1, k}: k=1, \ldots, N\right\}-$ по правилу

$$
Q_{t+1, k}= \begin{cases}\rho_{u}^{\prime}\left(z_{k}, u_{t}\right), & \text { если } k=k(t), \\ Q_{t, k} & \text { в противном случае. }\end{cases}
$$

Здесь $k=k(t)$ - индекс значения из $\left\{z_{1}, \ldots, z_{N}\right\}$, случайно выбранного на шаге $t$. Для сокращения вычислений лучше использовать следующее правило:

$$
Q_{t+1}=Q_{t}+\frac{1}{N}\left(\rho_{u}^{\prime}\left(z_{k}, u_{t}\right)-Q_{t, k}\right)
$$

Во всех алгоритмах параметр темпа обучения $\tau$ не зависит от номера шага.

Для улучшения сходимости можно применить схему усреднения градиаента, примененную в алгоритме AdaM (см. [8]). В ней применяется следующий метод обновления $u$ :

$$
m_{t+1}=\beta_{1} m_{t}+\left(1-\beta_{1}\right) Q_{t}, \quad v_{t+1}=\beta_{2} v_{t}+\left(1-\beta_{2}\right) Q_{t}^{2},
$$

$m_{t}$ и $v_{t}$-моменты первого и второго порядка, соответственно,

$$
\tilde{m}_{t+1}=\frac{m_{t+1}}{1-\beta_{1}^{t+1}}, \quad \tilde{v}_{t+1}=\frac{v_{t+1}}{1-\beta_{2}^{t+1}}
$$

$\tilde{m}_{t}$ и $\tilde{v}_{t}$-скорректированные значения моментов,

$$
u_{t+1}=u_{t}-\tau \frac{\tilde{m}_{t+1}}{\sqrt{\tilde{v}_{t+1}+\varepsilon}},
$$

$m_{0}=v_{0}=0 ; 0,5<\beta_{1} \leqslant \beta_{2}<1$. Эта схема позволяет повысить устойчивость процедуры поиска значения $u^{*}$, минимизирущего (11).

В тех случаях, когда имеется невырожденная вторая производная $\rho_{u u}^{\prime \prime}(z, u)$, можно также использовать метод Ньютона-Рафсона.

Итерационные процедуры вычисления значений стандартной усредняющей функции. При определенных условиях для вычисления среднего значения можно использовать следующий итерационный метод. Из определения следует, что значение $u=\mathrm{M}_{\rho}\left\{z_{1}, \ldots, z_{N}\right\}$ удовлетворяет следующему уравнению:

$$
\sum_{k=1}^{N} \rho^{\prime}\left(z_{k}-u\right)=0
$$

Введем обозначение $\varphi(x)=\rho^{\prime}(x) / x$ и предположим, что $\rho(x)$ дважды дифференцируема, а $\varphi(x)$ имеет смысл на всей области определения $\rho$. Тогда

$$
\sum_{k=1}^{N} \varphi\left(z_{k}-u\right) \cdot\left(z_{k}-u\right)=0
$$

откуда

$$
u=\sum_{k=1}^{N} \varphi\left(z_{k}-u\right) z_{k} / \sum_{k=1}^{N} \varphi\left(z_{k}-u\right) .
$$

Отсюда можно выписать итерционную схему вычисления $\bar{z}$ :

$$
u_{t+1}=\sum_{k=1}^{N} \varphi\left(z_{k}-u_{t}\right) z_{k} / \sum_{k=1}^{N} \varphi\left(z_{k}-u_{t}\right) .
$$


Данная схема сходится, если выполняется следующее условие:

$$
\left|\sum_{k=1}^{N} \varphi^{\prime}\left(z_{k}-\bar{z}\right)\left(z_{k}-\bar{z}\right)\right|<\left|\sum_{k=1}^{N} \varphi\left(z_{k}-\bar{z}\right)\right| .
$$

Например, для приведенных выше усредняющих агрегирующих функций $\mathrm{M}_{\alpha}$ и $\mathrm{M}_{\alpha}^{\gamma}$ при $0<\gamma<1$ это условие выполняется.

3. Принцип минимизации М-средних от параметризованных функций. Оптимальные значения параметров $\mathbf{w}^{*}$ теперь можно искать путем минимизации М-среднего от функций потерь:

$$
\mathcal{Q}(\mathbf{w})=\mathrm{M}_{\rho}\left\{\ell_{1}(\mathbf{w}), \ldots, \ell_{N}(\mathbf{w})\right\} .
$$

Если $\mathrm{M}_{\rho}$ имеет частные производные, то

$$
\operatorname{grad} \mathcal{Q}(\mathbf{w})=\sum_{k=1}^{N} v_{k}(\mathbf{w}, \bar{z}) \operatorname{grad} \ell_{k}(\mathbf{w}),
$$

где

$$
v_{k}(\mathbf{w}, \bar{z})=\frac{\partial \mathrm{M}_{\rho}\left\{\ell_{1}(\mathbf{w}), \ldots, \ell_{N}(\mathbf{w})\right\}}{\partial z_{k}} .
$$

Таким образом, градиент $\mathcal{Q}(\mathbf{w})$ является взвешенным средним арифметическим от градиентов $\ell_{1}(\mathbf{w}), \ldots, \ell_{N}(\mathbf{w})$.

Для минимизации $\mathcal{Q}$ можно было бы использовать любой из методов градиентного спуска, однако вычисление $v_{k}(\mathbf{w})$ может быть затратным из-за того, что для вычисления $\bar{z}$ необходимо решать задачу одномерной минимизации.

Для дальнейшего упрощения процедуры минимизации $\mathcal{Q}$ воспользуемся следующим фактом: оптимальные значения $\mathbf{w}^{*}$ и минимальное значение $u^{*}=\mathcal{Q}\left(\mathbf{w}^{*}\right)$ удовлетворяют следующей системе уравнений:

$$
\left\{\begin{array}{l}
\sum_{k=1}^{N} \rho_{u}^{\prime}\left(\ell_{k}(\mathbf{w})-u\right)=0, \\
\sum_{k=1}^{N} v_{k}(\mathbf{w}, u) \operatorname{grad} \ell_{k}(\mathbf{w})=0 .
\end{array}\right.
$$

Для ее решения можно воспользоваться следующим вариантом итерационного метода Якоби для решения систем нелинейных уравнений. На каждом шаге $t$ сначала решаем первое уравнение и находим значение

$$
u_{t}=\mathrm{M}_{\rho}\left\{\ell_{1}\left(\mathbf{w}_{t}\right), \ldots, \ell_{N}\left(\mathbf{w}_{t}\right)\right\} .
$$

Затем решаем систему уравнений

$$
\sum_{k=1}^{N} v_{k}\left(\mathbf{w}, u_{t}\right) \operatorname{grad} \ell_{k}(\mathbf{w})=0
$$

для поиска $\mathbf{w}_{t+1}$. Запишем ее на языке псевдокода:

$$
\begin{aligned}
& \text { procedure Yakoby }\left(\mathbf{w}_{0}\right) \\
& t \leftarrow 0 \\
& \text { repeat } \\
& \bar{z}_{t} \leftarrow \mathrm{M}_{\rho}\left\{\ell_{1}\left(\mathbf{w}_{t}\right), \ldots, \ell_{N}\left(\mathbf{w}_{t}\right)\right\} \\
& \mathbf{w}_{t+1} \leftarrow \mathbf{w}: \sum_{k=1}^{N} v_{k}\left(\mathbf{w}, \bar{z}_{t}\right) \operatorname{grad} \ell_{k}(\mathbf{w})=0 \\
& t \leftarrow t+1 \\
& \text { until }\left\{\bar{z}_{t}\right\} \text { и }\left\{\mathbf{w}_{t}\right\} \text { не стабилизируются }
\end{aligned}
$$


Для дальнейшего сокращения объема вычислений можно воспользоваться приемом, который был применен при построении алгоритма IRLS (iteratively reweighted least squares) для восстановления линейной регрессии при наличии выбросов. Так, в [17] была предложена процедура итеративно перевзвешенной минимизации эмпирического риска, которую назовем IR-ERM (iteratively reweighted empirical risk minimization). Она представляет собой итерационный процесс, в котором на каждом шаге $t$ сначала вычисляются компоненты градиента $v_{k}=v_{k}\left(\mathbf{w}_{t}, \bar{z}_{t}\right)$, а затем решается задача минимизации взвешенного эмпирического риска:

$$
\mathbf{w}_{t+1} \leftarrow \arg \min _{\mathbf{w}} \sum_{k=1}^{N} v_{k} \ell_{k}(\mathbf{w}) .
$$

На языке псевдокода процедуру целиком можно записать следующим образом:

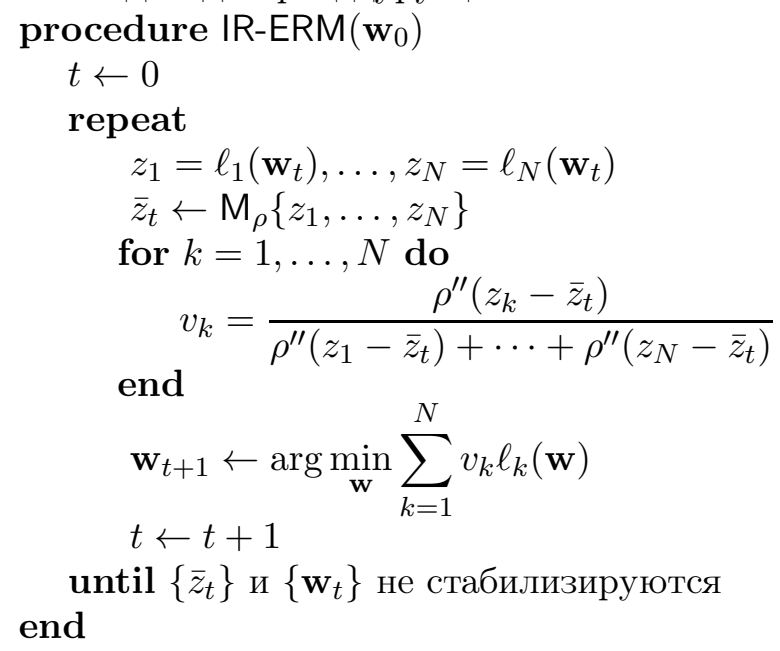

Для демонстрации возможности принципа минимизации М-средних и алгоритма IR-ERM по преодолению проблемы выбросов приведем пример восстановления линейной регрессии при наличии большого числа выбросов в данных.

Пример 1 (пример восстановления линейной регрессии). Данные представляют собой точки прямой с добавлением небольшого шума, распределенного равномерно, к которому добавляется относительно большое число больших выбросов ( $50 \%$ и $80 \%$ от первоначального объема данных, соответственно). Для восстановления применялся метод минимизации квадратов ошибок:

$$
\mathcal{Q}(\mathbf{w})=\frac{1}{N} \sum_{k=1}^{N} r_{k}^{2}(\mathbf{w}),
$$

более робастный метод минимизации абсолютных ошибок:

$$
\mathcal{Q}(\mathbf{w})=\frac{1}{N} \sum_{k=1}^{N}\left|r_{k}(\mathbf{w})\right|
$$

и метод минимизации М-среднего от квадратов ошибки на основе функции $\rho_{\varepsilon}(r)=\sqrt{\varepsilon^{2}+r^{2}}-\varepsilon$, где $\varepsilon=0,001$ :

$$
\mathcal{Q}(\mathbf{w})=\mathrm{M}_{\rho_{\varepsilon}}\left\{r_{1}^{2}(\mathbf{w}), \ldots, r_{N}^{2}(\mathbf{w})\right\} .
$$

На рис. 1 показаны результаты восстановления линейной регрессии. В обоих случаях метод минимизации М-среднего позволил избежать влияния выбросов, которого не удалось избежать при использовании принципа минимизации среднего как от квадратов, так и от абсолютных значений ошибок. 

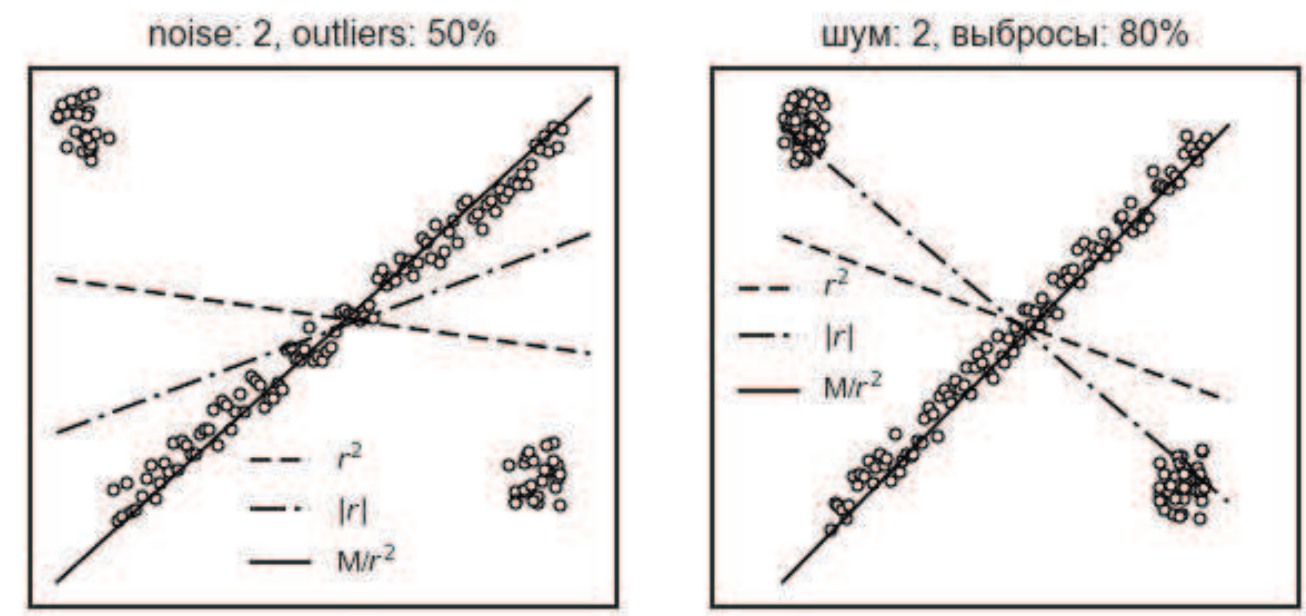

Рис. 1. Примеры восстановления линейной регрессии при наличии значительного числа выбросов в данных.

4. W-среднее арифметическое. В этом методе перед суммированием все значения, которые больше, чем заданное пороговое значение $u$, заменяются на $u$, т.е.

$$
\mathrm{WM}_{u}\left\{z_{1}, \ldots, z_{N}\right\}=\frac{1}{N} \sum_{k=1}^{N} \frac{1}{2}\left(z_{k}+u-\left|z_{k}-u\right|\right)
$$

назовем эту величину WM-средним арифметическим. Она обладает следующим свойством: если $u$-среднее арифметическое от $z_{1}, \ldots, z_{N}$, то

$$
\mathrm{WM}_{u}\left\{z_{1}, \ldots, z_{N}\right\}=\frac{z_{1}+\cdots+z_{N}}{N} .
$$

Обобщим понятие $\mathrm{W}$-среднего арифметического следующим образом.

Пусть $\mathrm{M}_{\rho}$ - робастное $\mathrm{M}$-среднее на базе дважды дифференцируемой строго выпуклой функции $\rho$, которое выбирается в качестве замены $\alpha$-квантиля. Она необходима для оценки значения параметра $u$. Введем обозначение $\bar{z}_{\rho}=\mathrm{M}_{\rho}\left\{z_{1}, \ldots, z_{N}\right\}$. Положим

$$
\mathrm{WM}_{\rho}\left\{z_{1}, \ldots, z_{N}\right\}=\frac{1}{N} \sum_{k=1}^{N} \frac{z+\bar{z}_{\rho}-\rho\left(z-\bar{z}_{\rho}\right)}{2} .
$$

Такой метод усреднения можно рассматривать как гладкий вариант W-среднего арифметического. В этой сумме используется гладкая замена значения слагаемого в окрестности порогового значения (см. рис. 2).

Вычислим частные производные:

$$
\frac{\partial \mathrm{WM}_{\rho}}{\partial z_{k}}=\frac{1}{2 N}\left(1-\rho^{\prime}\left(z_{k}-\bar{z}_{\rho}\right)\right)+\frac{1}{2 N} \frac{\partial \mathrm{M}_{\rho}}{\partial z_{k}}\left(N+\sum_{l=1}^{N} \rho^{\prime}\left(z_{l}-\bar{z}_{\rho}\right)\right) .
$$

По определению,

Поэтому

$$
\sum_{k=1}^{N} \rho^{\prime}\left(z_{k}-\bar{z}_{\rho}\right)=0
$$

$$
\frac{\partial \mathrm{WM}_{\rho}}{\partial z_{k}}=\frac{1}{2 N}\left(1-\rho^{\prime}\left(z_{k}-\bar{z}_{\rho}\right)\right)+\frac{1}{2} \frac{\partial \mathrm{M}_{\rho}}{\partial z_{k}}
$$




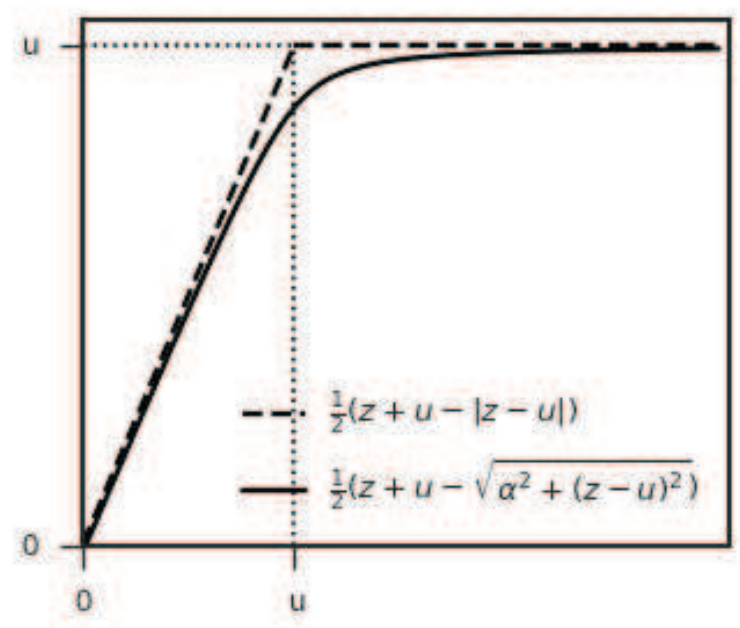

Рис. 2. Пример простого и гладкого усечения.

при этом

$$
\sum_{k=1}^{N} \frac{\partial \mathrm{WM}_{\rho}}{\partial z_{k}}=1
$$

5. Принцип минимизации W-среднего арифметического от потерь. Теперь можно рассмотреть задачу минимизации следующей функции среднего:

$$
\mathcal{Q}(\mathbf{w})=\mathrm{WM}_{\rho}\left\{\ell_{1}(\mathbf{w}), \ldots, \ell_{N}(\mathbf{w})\right\}
$$

для поиска оптимального набора параметров $\mathbf{w}^{*}$. Запишем градиент:

$$
\operatorname{grad} \mathcal{Q}(\mathbf{w})=\sum_{k=1}^{N} v_{k}(\mathbf{w}) \operatorname{grad} \ell_{k}(\mathbf{w}),
$$

где

a

$$
v_{k}(\mathbf{w})=\frac{1}{2 N}\left(1-\rho^{\prime}\left(\ell_{k}(\mathbf{w})-\bar{z}(\mathbf{w})\right)\right)+\frac{1}{2} \frac{\partial \mathrm{M}_{\rho}}{\partial z_{k}},
$$

$$
\bar{z}(\mathbf{w})=\mathrm{M}_{\rho}\left\{\ell_{1}(\mathbf{w}), \ldots, \ell_{N}(\mathbf{w})\right\} .
$$

Для численного расчета можно применить алгоритм IR-WERM (iteratively reweighted winsorized empirical risk), который является вариантом алгоритма итеративного перевзвешивания: procedure IR-WERM $\left(\mathbf{w}_{0}\right)$

$$
\begin{aligned}
& t \leftarrow 0 \\
& \text { repeat } \\
& \quad z_{1}=\ell_{1}\left(\mathbf{w}_{t}\right), \ldots, z_{N}=\ell_{N}\left(\mathbf{w}_{t}\right) \\
& \quad \bar{z}_{t} \leftarrow \mathrm{M}\left\{z_{1}, \ldots, z_{N}\right\} \\
& \quad \text { for } k=1, \ldots, N \text { do } \\
& \quad v_{k}=\frac{1}{2 N}\left(1-\rho^{\prime}\left(z_{k}-\bar{z}\right)\right)+\frac{1}{2} \frac{\partial \mathrm{M}_{\rho}}{\partial z_{k}} \\
& \quad \text { end } \\
& \quad \mathbf{w}_{t+1} \leftarrow \arg \min _{\mathbf{w}} \sum_{k=1}^{N} v_{k} \ell_{k}(\mathbf{w}) \\
& \quad t \leftarrow t+1 \\
& \text { until }\left\{\bar{z}_{t}\right\} \text { и }\left\{\mathbf{w}_{t}\right\} \text { стабилизируются }
\end{aligned}
$$
end 

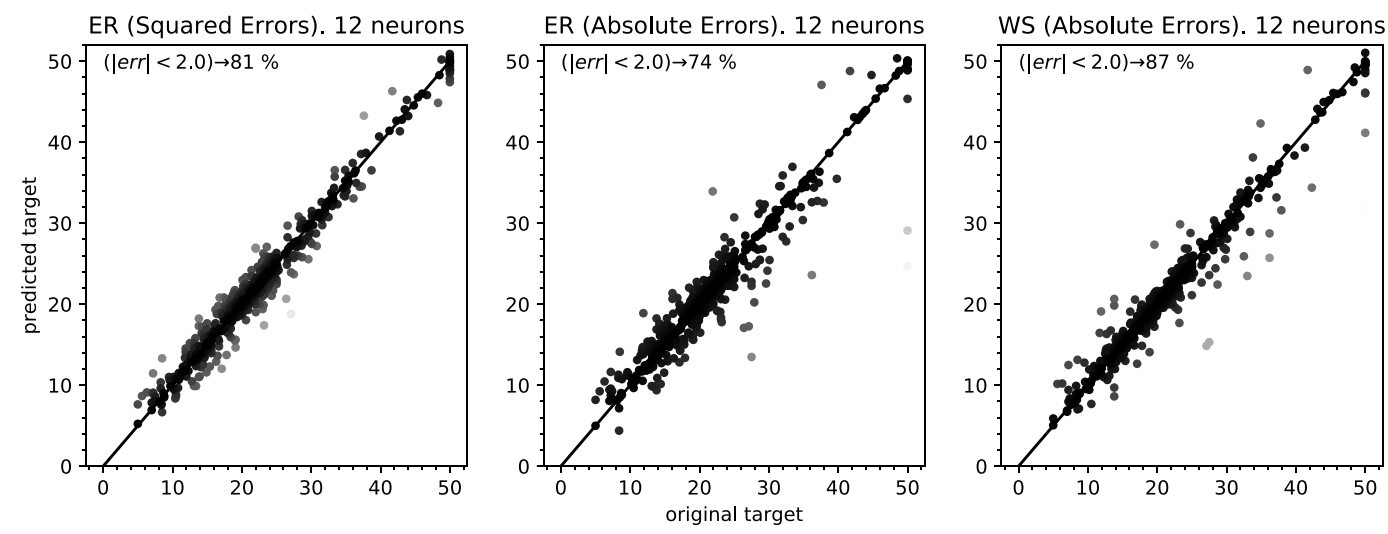

Рис. 3. Распределение ошибок обученной НС с одним скрытым слоем, содержащим 12 нейронов, на наборе данных Boston.

Пример 2 (обучение нейронной сети на наборе данных boston). Для иллюстрации возможностей алгоритма IR-WERM рассмотрим задачу обучения HC с одним скрытым слоем:

$$
\begin{aligned}
& y=w_{0}+w_{1} u_{1}+\cdots+w_{m} u_{m}, \\
& u_{j}=\sigma\left(w_{j 0}+w_{j 1} x_{1}+\cdots+w_{j n} x_{n}\right), \quad j=1, \ldots, m
\end{aligned}
$$

где

$$
\sigma(s)=\frac{1-e^{-s}}{1+e^{-s}} .
$$

Для обучения применялся метод обратного распространения ошибки, в котором минимизировалась средняя квадратичная ошибка ER и средняя абсолютная ошибка. Для обучения также применялся алгоритм IR-WERM, в котором минимизировалась робастная оценка суммы WS квадратов ошибки WS с функцией $\rho_{\varepsilon, \alpha}$ вида $(10)$, где $\rho_{\varepsilon}(r)=\sqrt{\varepsilon^{2}+r^{2}}-\varepsilon, \varepsilon=1, \alpha=0,80$. На рис. 3 представлены распределения точек с координатами ( $\left.y_{\text {orig }}, y_{\text {pred }}\right)$, где $y_{\text {orig }}$ - известное значение, $y_{\text {pred }}$ - значение, вычисленное при помощи обученной НС. Они наглядно демонстрируют, что при обучении НC при помощи алгоритма IR-WERM уменьшаются величины ошибок на более чем $80 \%$ данных.

Следующие два примера основаны на искусственных наборах данных, сгенерированных по схеме, описанной в [3]. Каждый пример представляет данные для восстановления некоторой функции $f(\mathbf{x})$, где $\mathbf{x} \in \mathbb{R}^{3}$. Обучающие и тестовые данные по 1000 элементов выбраны случайно в соответствии с равномерным случайным распределением на $[-2,2]^{3}$. Они являются зашумленными равномерно распределенным шумом на $[-0,2 ; 0,2]$. В обучающие наборы данных добавлены выбросы $30 \%$ и $50 \%$ соответственно; в тестовых наборах данных выбросов нет. Каждый выбросэто замена ожидаемого значения на значение 20. Для каждого примера приведен рисунок, на котором изображены распределения точек с координатами $\left(y_{\text {orig }}, y_{\text {pred }}\right)$, где $y_{\text {orig }}$ - известное значение из набора данных без выбросов, $y_{\text {pred }}$ - значение, вычисленное при помощи НС, обученной на наборе данных с выбросами.

Пример 3. $f(\mathbf{x})=\|\mathbf{x}\|^{2 / 3}$ (см. рис. 4).

Пример 4. $f(\mathbf{x})=\sin \|\mathbf{x}\| /\left(\|\mathbf{x}\|+10^{-8}\right)$ (см. рис. 5).

6. От М-среднего арифметического к WM-средним. Запишем W-среднее арифметическое в следующем виде:

$$
\operatorname{WM}\left\{z_{1}, \ldots, z_{N}\right\}=\frac{1}{N} \sum_{k=1}^{N} \sigma\left(z_{k}, z^{*}\right)
$$



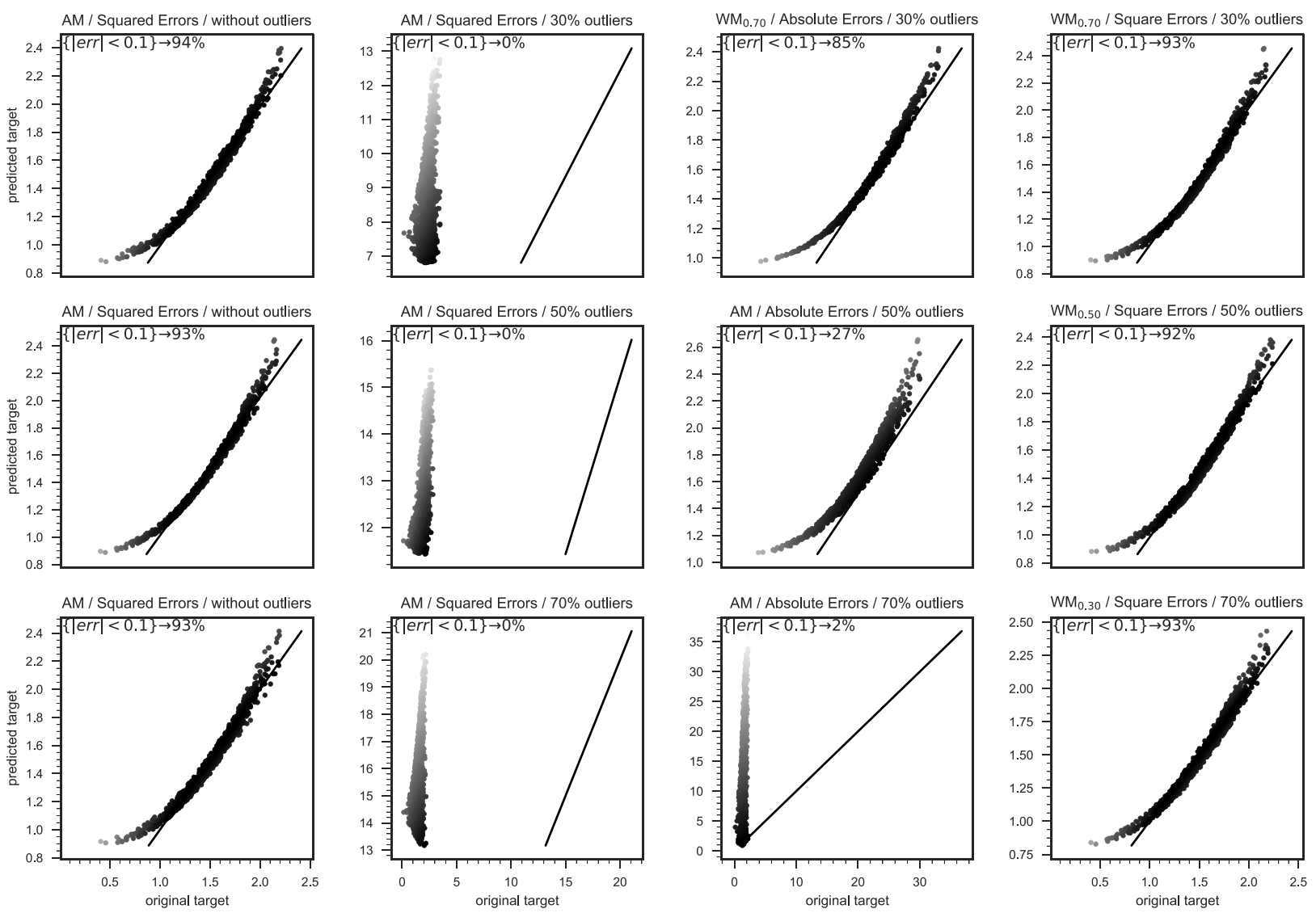

Рис. 4. Распределение ошибок обученной НС с одним скрытым слоем, содержащим 12 нейронов, на наборе данных из примера 3.

где

$$
\sigma\left(z, z^{*}\right)=\min \left\{z, z^{*}\right\} .
$$

WM - это частный случай М-среднего:

$$
\mathbf{M}_{\rho}\left\{\sigma\left(z_{1}, z^{*}\right), \ldots, \sigma\left(z_{N}, z^{*}\right)\right\}
$$

когда $\rho(r)=r^{2}$.

Так как основная проблема в том, чтобы научиться отсекать слишком большие значения в распределении потерь $z_{(1)}, \ldots, z_{(N)}$ от ошибок в задаче регрессии или от отступа в задаче классификации, то можно было бы положить $z^{*}=\mathrm{M}_{\chi}\left\{z_{1}, \ldots, z_{N}\right\}$, где $\mathrm{M}_{\chi}$ - это квантиль $\mathrm{c} \alpha \geqslant 0,5$, но лучше использовать его непрерывно дифференцируемые аналоги. Исходя из этого определим Мсреднее

$$
\mathrm{WM}_{\rho \chi}\left\{z_{1}, \ldots, z_{N}\right\}=\arg \min _{u} \sum_{k=1}^{N} \rho\left(\sigma\left(z_{k}, \bar{z}_{\chi}\right)-u\right),
$$

где $\bar{z}_{\chi}=\mathrm{M}_{\chi}\left\{z_{1}, \ldots, z_{N}\right\}$. Тогда $\bar{z}_{\rho \chi}=\mathrm{M}_{\rho \chi}\left\{z_{1}, \ldots, z_{N}\right\}$ является решением уравнения:

$$
\sum_{k=1}^{N} \rho^{\prime}\left(u_{k}-\bar{z}_{\rho \chi}\right)=0
$$

где $u_{k}=\sigma\left(z_{k}, \bar{z}_{\chi}\right)$. Частная производная $\partial \mathrm{M}_{\rho \chi} / \partial z_{k}$ является решением уравнения

$$
\rho^{\prime \prime}\left(u_{k}-u\right) \sigma_{z}^{\prime}\left(z_{k}, \bar{z}_{\chi}\right)+\frac{\partial \mathrm{M}_{\chi}}{\partial z_{k}} \sum_{l=1}^{N} \rho^{\prime \prime}\left(u_{l}-u\right) \sigma_{z^{*}}^{\prime}\left(z_{l}, \bar{z}_{\chi}\right)-\frac{\partial \mathrm{WM}_{\rho \chi}}{\partial z_{k}} \sum_{l=1}^{N} \rho^{\prime \prime}\left(u_{l}-u\right)=0 .
$$



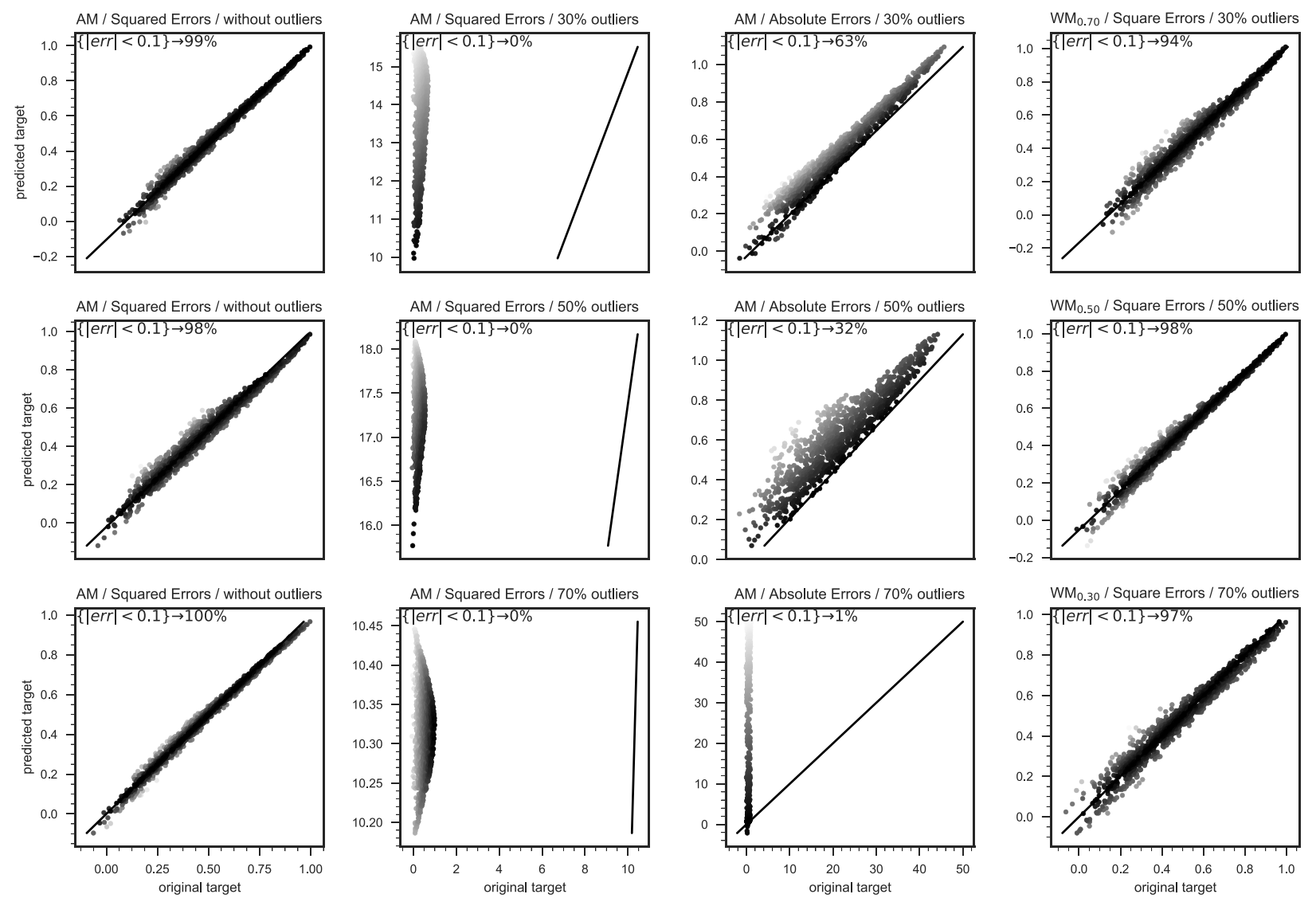

Рис. 5. Распределение ошибок обученной НС с одним скрытым слоем, содержащим 12 нейронов, на наборе данных из примера 4.

Отсюда

$$
\frac{\partial \mathrm{WM}_{\rho \chi}}{\partial z_{k}}=\frac{\partial \mathbf{M}_{\rho}}{\partial u_{k}} \sigma_{z}^{\prime}\left(z_{k}, \bar{z}_{\chi}\right)+\frac{\partial \mathbf{M}_{\chi}}{\partial z_{k}} \mu
$$

где

$$
\mu=\sum_{l=1}^{N} \frac{\partial \mathrm{M}_{\rho}}{\partial u_{l}} \sigma_{z^{*}}^{\prime}\left(z_{l}, \bar{z}_{\chi}\right) .
$$

Приведем примеры для некоторых функций $\sigma\left(z, \bar{z}_{\chi}\right)$.

1. Пусть $\sigma_{\chi}\left(z, \bar{z}_{\chi}\right)=\min \left\{z, \bar{z}_{\chi}\right\} ;$ тогда

$$
\frac{\partial \mathbf{W M}_{\rho \chi}}{\partial z_{k}}= \begin{cases}\frac{\partial \mathbf{M}_{\rho}}{\partial z_{k}}+\frac{\partial \mathbf{M}_{\chi}}{\partial z_{k}} \sum_{l=m+1}^{N} \frac{\partial \mathbf{M}_{\rho}}{\partial z_{l}}, & \text { если } k \leqslant m, \\ \frac{\partial \mathbf{M}_{\chi}}{\partial z_{k}} \sum_{l=m+1}^{N} \frac{\partial \mathbf{M}_{\rho}}{\partial z_{l}}, & \text { если } k>m .\end{cases}
$$

В частности, если $\mathrm{M}_{\rho}$ - среднее арифметическое, то

$$
\frac{\partial \mathrm{WM}_{\rho \chi}}{\partial z_{k}}= \begin{cases}\frac{1}{N}+\frac{N-m}{N} \frac{\partial \mathrm{M}_{\chi}}{\partial z_{k}}, & \text { если } k \leqslant m, \\ \frac{N-m}{N} \frac{\partial \mathrm{M}_{\chi}}{\partial z_{k}}, & \text { если } k>m .\end{cases}
$$


2. Пусть $\sigma_{\chi}\left(z, \bar{z}_{\chi}\right)=\frac{1}{2}\left(z+\bar{z}_{\chi}-\chi\left(z-\bar{z}_{\chi}\right)\right)$; тогда

$$
\frac{\partial \mathbf{W M}_{\rho \chi}}{\partial z_{k}}=\frac{\partial \mathrm{M}_{\rho}}{\partial u_{k}} \frac{1-\chi^{\prime}\left(z_{k}-\bar{z}_{\chi}\right)}{2}+\frac{\partial \mathrm{M}_{\chi}}{\partial z_{k}} \sum_{l=1}^{N} \frac{\partial \mathrm{M}_{\rho}}{\partial u_{l}} \frac{1+\chi^{\prime}\left(z_{l}-\bar{z}_{\chi}\right)}{2}
$$

3. Пусть $\sigma_{\chi}\left(z, \bar{z}_{\chi}\right)=-\frac{1}{\lambda} \ln \left(e^{-\lambda z}+e^{-\lambda \bar{z}_{\chi}}\right)$ (мягкий минимум); тогда

$$
\frac{\partial \mathbf{W M}_{\rho \chi}}{\partial z_{k}}=\frac{\partial \mathbf{M}_{\rho}}{\partial u_{k}} \frac{1}{1+e^{\lambda\left(z_{k}-\bar{z}_{\chi}\right)}}+\frac{\partial \mathrm{M}_{\chi}}{\partial z_{k}} \sum_{l=1}^{N} \frac{\partial \mathrm{M}_{\rho}}{\partial u_{l}} \frac{1}{1+e^{-\lambda\left(z_{l}-\bar{z}_{\chi}\right)}} .
$$

\section{СПИСОК ЛИТЕРАТУРЫ}

1. Хъюбер П. Робастность в статистике. - Мир, 1984.

2. Шибзухов 3. М. Агрегирующие корректные операции над алгоритмами// Докл. РАН. - 2015. - 91, № 3. - C. 391-393.

3. Beliakov G., Kelarev A., Yearwood J. Robust artificial neural networks and outlier detection/ arxiv:1110.0169v1 [math.0C].

4. Beliakov G. , Sola H., Calvo T. A Practical Guide to Averaging Functions. - Springer, 2016.

5. Calvo T., Beliakov G. Aggregation functions based on penalties// Fuzzy Sets Syst. — 2010. — 161, № 10. - P. $1420-1436$.

6. Grabich M., Marichal J.-L., Pap E. Aggregation Functions. - Cambridge: Cambridge Univ. Press, 2009.

7. Huber P. J. Robust Statistics. - New York: Wiley, 1981.

8. Knigma D. P., Ba J. Adam: A Method for Stochastic Optimization/ arxiv:1412.6980.

9. Koenker R. Quantile regression. - New York: Campridge Univ. Press, 2005.

10. Ma Y., Li L., Huang X., Wang S. Robust support vector machine using least median loss penalty// IFAC Proc. Vols. - 2011. - 44, № 1. - P. 11208-11213.

11. Mesiar R., Komornikova M., Kolesarova A., Calvo T. Aggregation functions: A revision// in: Fuzzy Sets and Their Extensions: Representation, Aggregation and Models (Bustince H., Herrera F., Montero J., eds.). - Berlin-Heidelberg: Springer, 2008.

12. Newey W., Powell J. Asymmetric least squares estimation and testing// Econometrica. — 1987. — 55, № 4. - P. 819-847.

13. Rousseeuw P. J. Least median of squares regression// J. Am. Stat. Ass. — 1984. — № 79. — P. 871-880.

14. Rousseeuw P. J., Leroy A. M. Robust regression and outlier detection. - New York: Wiley, 1987.

15. Schmidt M., Le Roux N., Bach F. Minimizing finite sums with the stochastic average gradient// Math. Program. - 2017. - 162, № 1-2. - P. 83-112.

16. Shibzukhov Z. M. Correct aggregate operations with algorithms// Pattern Recogn. Image Anal. — 2014. - 24, № 3. - P. 377-382.

17. Shibzukhov Z. M. On the principle of empirical risk minimization based on averaging aggregation functions// Dokl. Math. - 2017. — 96, № 2. - P. 494-497.

18. Vapnik V. The Nature of Statistical Learning Theory. — Springer-Verlag, 2000.

19. Yohai V. J. High breakdown-point and high effciency robust estimates for regression// Ann. Stat. — 1987. — № 15. - P. 642-656.

\section{Шибзухов Заур Мухадинович}

Институт прикладной математики и автоматизации,

Кабардино-Балкарский научный центр Российской академии наук, Нальчик

E-mail: szport@gmail.com 